\title{
Effects of donepezil on behavioral manifestations of thalamic infarction: a single-case observation
}

\author{
Rodrigo Riveros ${ }^{1,2}$, Hughes Chabriat ${ }^{3}$, Rodrigo Flores ${ }^{4}$, Gonzalo Alvarez $^{5}$ and Andrea Slachevsky ${ }^{1,6,78 *}$ \\ ${ }^{1}$ Cognitive Neurology and Dementia Unit, Neurology Service, El Salvador Hospital, Santiago, Chile \\ ${ }^{2}$ School of Psychology, Universidad Mayor, Santiago, Chile \\ ${ }^{3}$ Department of Neurology, Hopital Lariboisière, Paris, France \\ ${ }^{4}$ Neuroradiology Service, Instituto de Neurocirugía Dr. Asenjo, Santiago, Chile \\ ${ }^{5}$ Neurology Service, Hospital de El Salvador Santiago, Santiago, Chile \\ ${ }^{6}$ Molecular and Clinical Pharmacology, Institute of Biomedical Sciences, Faculty of Medicine, Universidad de Chile, Santiago, Chile \\ 7 Faculty of Medicine, Department of Neurological Sciences, Universidad de Chile, Santiago, Chile \\ ${ }^{8}$ Centre of Advanced Research in Education, Universidad de Chile, Santiago, Chile
}

\section{Edited by:}

Oscar López, University of Pittsburgh

School of Medicine, USA

\section{Reviewed by:}

Oscar López, University of Pittsburgh

School of Medicine, USA

Mario Riverol, Clinica Universidad de

Navarra, Spain

${ }^{*}$ Correspondence:

Andrea Slachevsky, Centre of

Advanced Research in Education,

Universidad de Chile, Periodista José

Carrasco Tapia $n^{\circ} 75$, Santiago, Chile.

e-mail: aslachevsky@me.com
Objective: To examine the effect of donepezil for the treatment of cognitive and behavioral disorders associated with thalamic lesions in a 45-year-old male who suffered an infarct in the left thalamus. Background: Recent studies suggest that donepezil may improve executive functions impairments due to subcortical ischemic lesions. Method: The effects of donepezil were analyzed in a single-case of thalamic infarction with cognitive and behavioral alterations in an open label study. Results: Significant behavioral modifications related to improved performances in executive functions were observed with the treatment. Conclusion: The results suggest that donepezil may have significant effect on executive functions that can alter behavioral outcomes after thalamic infarctions.

\footnotetext{
Keywords: donepezil, vascular dementia, vascular subcortical cognitive impairment, thalamus, dysexecutive syndrome, stroke
}

\section{INTRODUCTION}

Thalamic infarcts can be responsible for cognitive, emotional and/or behavioral alterations with significant impact on activities of daily life (Roman et al., 2005). Donepezil is a cholinesterase inhibitor currently approved for the treatment of Alzheimer's Disease that may also provide some benefits in vascular dementia and vascular cognitive impairment (Erkinjuntti et al., 2004). Despite the absence of appropriate evaluation of executive functions in previous vascular dementia trials, the results of a recent placebo-controlled study performed in demented as well as in nondemented CADASIL patients strongly support that donepezil may mainly improve executive functions in the presence of subcortical ischemic lesions (Dichgans et al., 2008). Benefits of cholinesterase inhibitors on behavior and cognition have been also reported on other conditions that can affect cholinergic pathways connecting cortical and subcortical structures, such as traumatic brain injury (Tenovuo, 2005). Moreover, donepezil has also been suggested for the treatment of depression and apathy, both symptoms being, at least, partially modulated by cholinergic innervations (Whyte et al., 2008). All these conditions affect cholinergic nuclei like the basal forebrain, thalamus, and their innervations to the cerebral cortex (Tenovuo, 2005). These findings are consistent with neuro-anatomical evidence and clinical trials suggesting that cholinergic pathways are involved in cognition and neuropsychiatric symptoms, especially apathy (Selden et al., 1998; Reynolds et al., 2011).

Damage to the frontal cortex and to the subcortical related structures are presumably responsible for the cognitive and behavioral symptoms gathered into the so-called "dysexecutive syndrome" (DS; Chaytor and Schmitter-Edgecombe, 2007). The DS can be analyzed according to its cognitive and behavioral components using dedicated tests (Chaytor and Schmitter-Edgecombe, 2007). Structured and reliable questionnaires have also been recently developed to assess the manifestations of DS in daily life since there is only a weak relationship between the results of neuropsychological testing and the daily life consequences of this syndrome. This discrepancy is presumably related to the lack of behavioral evaluation in the neuropsychological testing (Chaytor and Schmitter-Edgecombe, 2007). The Dysexecutive Questionnaire (DEX) is a 20-item questionnaire that uses a 5-point Likert scale to assess the dysexecutive symptoms. The DEX scores range from 0 to 80 points, with higher scores reflecting higher difficulties in daily life. The DEX questionnaire belongs to the "Behavioral Assessment of DS," battery and describes with fair confidence the non-routine everyday problems arising from executive dysfunction (Wilson et al., 1998).

We report a single-case with a left thalamic infarction and behavioral disturbances that was treated with donepezil for 6 months using a open label study with an off-on-off design. As an effect of the treatment, we hypothesized an improvement of dysexecutive symptoms and apathy.

\section{CASE REPORT}

L.G. was a 45-year-old male, right-handed, with no history of neurological or psychiatric disease. He worked as a supermarket store manager. He presented an amnesic syndrome and behavioral disturbances of sudden onset in the absence of focal signs at neurological examination. 
These clinical manifestations occurred with an isolated infarction located on magnetic resonance imaging (MRI) in the ventrolateral and ventroanterior nucleus of the left thalamus (Figure 1). He received a full etiology study that did not reveal any etiology. Since this event, the patient was unable to resume his previous work and only sustained temporary jobs. The patient and his wife

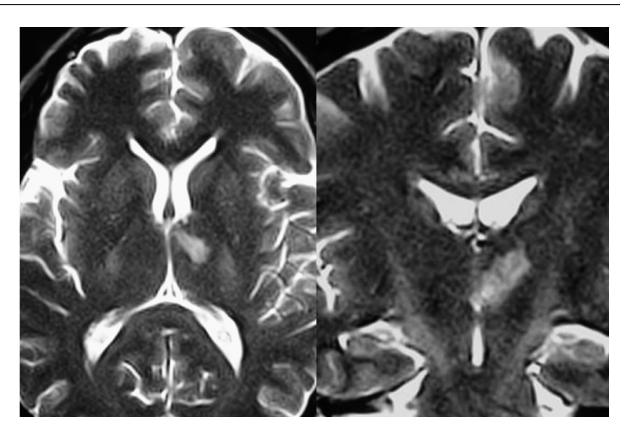

FIGURE 1 | Brain Nuclear Magnetic Resonance. T2 DWI axial (left) and coronal (right) views of the patient showing left thalamic infarction in the anterior nuclei, ventral lateral nuclei and anterior part of the median dorsal nuclei. The mamillo-thalamic tract and internal medullary lamina are also compromised. No other ischemic lesions were found. complained about memory disturbances and inability to carry on his job. His wife also referred behavior disturbances, mainly apathy, and difficulty to plan. He did refer neither depressive symptoms, nor anxiety.

Two years later, he was first evaluated by neuropsychological testing. Both the patient and his wife confirmed that there was no significant change in his cognitive functioning over the two previous years. The global cognitive performances were assessed using the mini-mental state examination (MMSE; Folstein et al., 1975), the working memory using the Digit Span and the verbal learning capabilities using the free and cued selective reminding test (FCSRT; Grober et al., 2008). The examination of executive functions was carried out using the Frontal Assessment at Bedside (Dubois et al., 2000), the category and literal verbal fluency tests and the modified version of Wisconsin card sorting test (MWCST; Van den Broek et al., 1993). The everyday memory difficulties were evaluated using the Mac Nair's Cognitive Difficulties Scale, which was applied to both the patient and his wife (Derouesne et al., 1993). The latter was also questioned using the neuropsychiatric inventory (NPI) and the Alzheimer's Disease Cooperative Study-Activities of Daily Living Inventory (ADCS-ADL; Cummings et al., 1994; Galasko et al., 1997). Finally, the dysexecutive behavior in everyday life was assessed using the DEX questionnaire applied to the patient's wife (Wilson et al., 1998).

Table 1 | Patient scores in cognitive battery and behavioral questionnaires for baseline, 3 and 6 months of treatment and 3 months after treatment discontinuation.

\begin{tabular}{|c|c|c|c|c|}
\hline Test (max. test score) & Baseline & 3 Months & 6 Months & $\begin{array}{l}3 \text { Months } \\
\text { after discontinuation }\end{array}$ \\
\hline
\end{tabular}

Global cognitive efficiency

MMSE (/30)

Attention and working memory

Direct digit span (/7)

Reverse digit span (/7)

Episodic memory

Verbal learning (selective reminding test Grober and Buschke test)

Immediate cue recall (/16)

Sum of free and cued recall (/48)

Intrusions

Sum of free and cued delayed recall (/16)

Warrington's recognition memory faces (/50)

Executive functions

Verbal fluency FAS*

Wisconsin card sorting test

Categories (/6)

Perseverative errors

Neuropsychiatric evaluation

NPI* total score (/144)

NPI apathy item - frequency (/4)

$\mathrm{NPI}$ apathy item - severity (/3)

NPI apathy item - stress (/5)

Dysexecutive Questionnaire (/80)

ADCS-ADL $(/ 100)^{* *}$

Cognitive difficulties scale Mac Nair (/80)
26

5

4

12

21

3

3

48

9.7 (2.5)

6

1

84

4

2

3

42

62

43
27

5

4

15

19

12

5

NA

$13.3(2.1)$

5

4

72

2

1

2

27

70

30
27

4

5

16

20

4

8

NA

$10.7(0.6)$

6

3

90

1

1

0

17

76

31
28

5

4

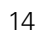

29

6

9

NA

$12.7(2.1)$

3

8

140

3

2

3

35

77

29

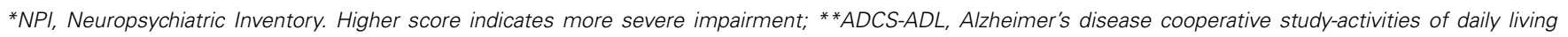
inventory. Higher score indicates less impairment. 
The neuropsychological evaluation revealed a reduced global cognitive capacity, impairment in episodic memory and a DS with a reduced verbal fluency and cognitive flexibility associated with impaired conceptualization (Table $\mathbf{1}$ ).

We then proposed to the patient an open crossover study using donepezil to assess whether this treatment could actually modify his cognitive status. The study was approved by the local Ethics Committee (El Salvador Hospital). An informed consent was obtained from the patient and from his wife for the trial.

The drug was administered daily for 6 months (donepezil $5 \mathrm{mg} /$ daily during the first month, and $10 \mathrm{mg} /$ daily during the next 5 months). The patient was assessed at baseline before treatment and at 3 and 6 months under treatment using the same neuropsychological tests. A final evaluation was carried out 3 months after discontinuation of the drug (Table 1). The questionnaires were applied to the patient and to his wife during the same visits.

The drug was well tolerated and no adverse event was reported by the patient. No significant change was detected on the performances of the different neuropsychological tests including those sensitive to executive dysfunction such as the Frontal Assessment at Bedside, the FCSRT and the MWCST.

In contrast, significant changes were detected by the patient and his wife in the behavioral outcomes assessed by the DEX questionnaire and by the NPI severity and stress scores. "The DEX scores improved during the treatment relative to baseline and worsened after donepezil discontinuation. Total DEX scores were 42,27 , and 17 points at baseline, 3 and 6 months of treatment, respectively. Subsequently, symptoms were increased when medication was discontinued (total DEX score: 35 ). The NPI apathy scores also improved under treatment and worsened with medication discontinuation. This was observed for apathy on both severity and stress sub-scores (see Figure 2; Table 1).”
The patient's wife particularly reported that under the treatment, the patient was much less apathetic, was more attentive, had more initiative, and returned to working activities (he was employed as gained employment as an auto repair center manager while under donepezil).

\section{DISCUSSION}

The cognitive and behavioral disturbances in the present observation have been repeatedly reported in association with thalamic infarctions. Verbal amnesia has been particularly associated with lesions involving the anterior nucleus and mamillo-thalamic tract of the left thalamus, dysexecutive symptoms with thalamic lesions involving the mediodorsal nucleus and/or internal medullary lamina and apathy with lesions in the anterior thalamic nucleus (Van der Werf et al., 2003; Carrera and Bogousslavsky, 2006).

In this open label study, donepezil seems to have some ability to modulate persisting behavioral manifestations at the late stage of an isolated left-sided thalamic infarction. As we expected, executive performance and neuropsychiatric symptoms were improved during treatment.

Measurable effects were detected under the treatment period using the DEX questionnaire and the apathy sub-scores of the NPI while no significant changes in the other neuropsychological tests were detected. These effects were reversed after discontinuation of the cholinesterase inhibitor. These behavioral changes were also detected in the absence of significant modifications of the MMSE score and of episodic memory performances in line with the poor effects of donepezil on global cognitive or memory performances in previous vascular dementia trials (Erkinjuntti et al., 2004; Roman et al., 2005; Dichgans et al., 2008). In this study, not only the NPI, that is largely used for the assessment of behavioral alterations, but also the DEX questionnaire was applied to assess the possible changes under donepezil. The DEX

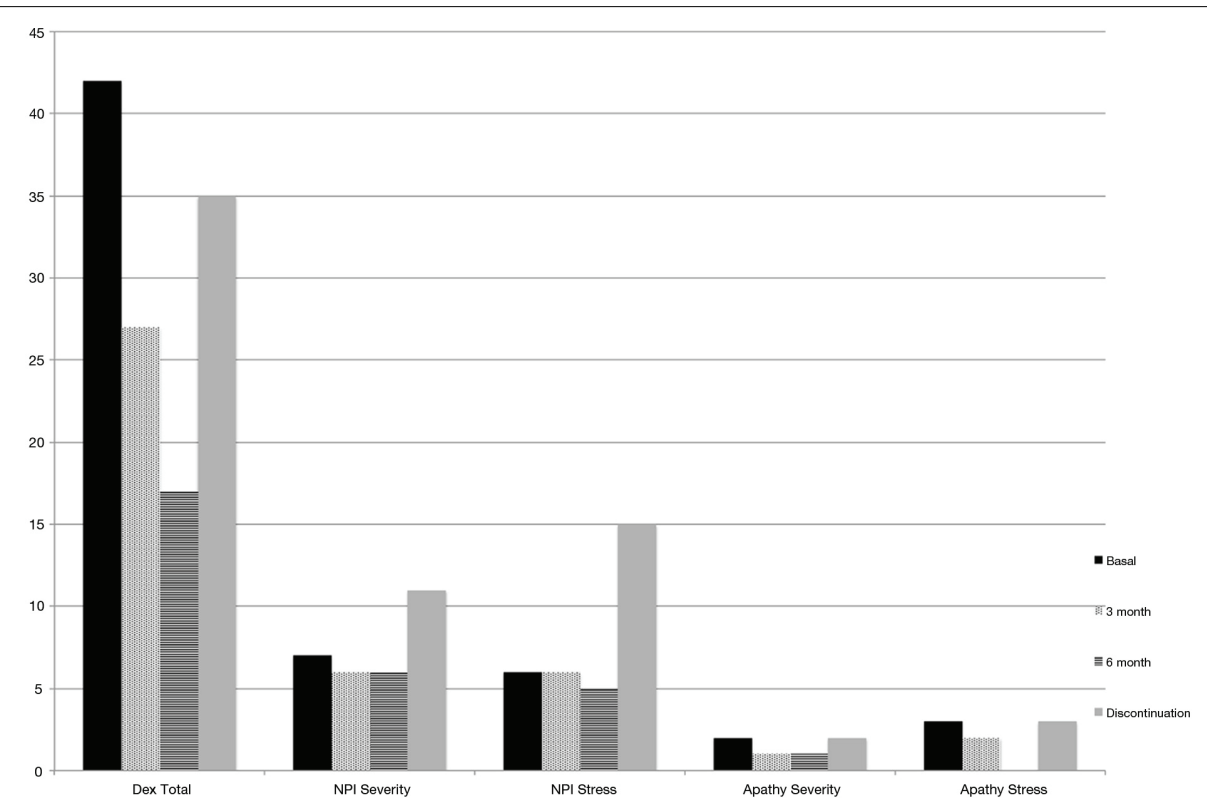

FIGURE 2 | Dysexecutive Questionnaire Score and NPI Scores for Apathy item. Performance is improved along treatment; however it is worsened when drug intake is discontinued. 
questionnaire provides a number of advantages over the clinical measures of executive functions. It is not limited to the evaluation of the cognitive aspects of the DS but it also includes the evaluation of its emotional, motivational, and behavioral components. The questionnaire is easy to administer and includes a self-reported as well as an informant-reported part specifically designed to assess the participant's behavior in daily life. Its psychometric properties on ecological and discriminative validity have been already documented (Wilson et al., 1998).

This single-case study is a limited, preliminary clinical experience of the use of donepezil in behavioral disturbances associated with an isolated thalamic infarction. This case study has obvious limitations. Some fluctuations of the data obtained using questionnaires, placebo effects related to the intake of a new drug in an isolated case or multiple biases related to the non-blinded treatment may cause that the improvement of behavior under treatment is only detected by chance in this observation. In contrast, the importance of the effect with actual consequences in daily life, the variations of the DEX scores much larger than those reported after its repeated administration (Shinagawa et al., 2007), the congruence of the observed effects in the present case with data on cholinesterase inhibitors in the literature, and its partial reversibility support that donepezil was actually efficient and may have real effects on behavior and mainly on apathy after isolated thalamic lesions (Erkinjuntti et al., 2004; Dichgans et al., 2008).

In conclusion, the capacity of donepezil to modulate some behavioral consequences of subcortical lesions, particularly those related to thalamic lesions, as suggested by this observation, needs further research. Although little is known about cholinesterase inhibitors in vascular dementia and being this is a single-case study, our results encourage research in groups of patients. The present data suggests that future studies should not only focus on the cognitive effects of cholinesterase inhibitors but also on its behavioral effects, especially in daily life, using new and dedicated tools for this purpose.

\section{ACKNOWLEDGMENTS}

This study was funded by PIA-CONICYT, Proyect CIE-05.

\section{REFERENCES}

Carrera, E., and Bogousslavsky, J. (2006). The thalamus and behavior: effects of anatomically distinct strokes. Neurology 66, 1817-1823.

Chaytor, N., and Schmitter-Edgecombe, M. (2007). Fractionation of the dysexecutive syndrome in a heterogeneous neurological sample: comparing the Dysexecutive Questionnaire and the Brock Adaptive Functioning Questionnaire. Brain Inj. 21, 615-621.

Cummings, J. L., Mega, M., Gray, K., Rosenberg-Thompson, S., Carusi, D. A., and Gornbein, J. (1994). The Neuropsychiatric Inventory: comprehensive assessment of psychopathology in dementia. Neurology 44, 2308-2314.

Derouesne, C., Dealberto, M., and Boyer, P. (1993). Empirical evaluation of the Cognitive Difficulties Scale for assessment of memory complaints in general practice: a study of 1628 cognitively normal subjects aged 45-75 years. Int. J. Geriatr. Psychiatry 8, 599-607.

Dichgans, M., Markus, H. S., Salloway, S., Verkkoniemi, A., Moline, M., Wang, Q., Posner, H., and Chabriat, H. S. (2008). Donepezil in patients with subcortical vascular cognitive impairment: a randomised doubleblind trial in CADASIL. Lancet Neurol. 7, 310-318.

Dubois, B., Slachevsky, A., Litvan, I., and Pillon, B. (2000). The FAB: a frontal assessment battery at bedside. Neurology 55, 1621-1626.

Erkinjuntti, T., Roman, G., and Gauthier, S. (2004). Treatment of vascular dementia-evidence from clinical trials with cholinesterase inhibitors. Neurol. Res. 26, 603-605.

Folstein, M. F., Folstein, S. E., and McHugh, P. R. (1975). "Mini-mental state". A practical method for grading the cognitive state of patients for the clinician. J. Psychiatr. Res. 12, 189-198.

Galasko, D., Bennett, D., Sano, M., Ernesto, C., Thomas, R., Grundman, M., and Ferris, S. (1997). An inventory to assess activities of daily living for clinical trials in Alzheimer's disease. The Alzheimer's Disease Cooperative Study. Alzheimer Dis. Assoc. Disord. 11(Suppl. 2), S33-S39.

Grober, E., Hall, C. B., Lipton, R. B., Zonderman, A. B., Resnick, S. M., and Kawas, C. (2008). Memory impairment, executive dysfunction, and intellectual decline in preclinical Alzheimer's disease. J. Int. Neuropsychol. Soc. 14, 266-278.

Reynolds, C., Butters, M., Lopez, O., Pollock, B., Dew, M., Mulsant, B., Lenze, E., Holm, M., Rogers, J., Mazumdar, S., Houck, P., Begley, A., Anderson, S., Karp, J., Miller, M., Whyte, E., Stack, J., Gildengers, A., Szanto, K., Bensasi, S., Kaufer, D., Kamboh, M., and DeKosky, S. (2011). Maintenance treatment of depression in old age: a randomized, double-blind, placebo-controlled evaluation of the efficacy and safety of donepezil combined with antidepressant pharmacotherapy. Arch. Gen. Psychiatry 68, 51-60.

Roman, G. C., Wilkinson, D. G., Doody, R. S., Black, S. E., Salloway, S. P., and Schindler, R. J. (2005). Donepezil in vascular dementia: combined analysis of two large-scale clinical trials.
Dement. Geriatr. Cogn. Disord. 20, 338-344.

Selden, N., Gitelman, D., SalamonMurayaman, N., Parrish, T., and Mesulam, M. (1998). Trajectories of cholinergic pathways within the cerebral hemispheres of the human brain. Brain 121, 2249-2257.

Shinagawa, Y., Nakaaki, S., Hongo, J., Murata, Y., Sato, J., Matsui, T., Tatsumi, H., Akechi, T., and Furukawa, T. A. (2007). Reliability and validity of the Japanese version of the Dysexecutive Questionnaire (DEX) in Alzheimer's disease: validation of a behavioral rating scale to assess dysexecutive symptoms in Japanese patients with Alzheimer's disease. Int. J. Geriatr. Psychiatry 22, 951-956.

Tenovuo, O. (2005). Central acetylcholinesterase inhibitors in the treatment of chronic traumatic brain injury-clinical experience in 111 patients. Prog. Neuropsychopharmacol. Biol. Psychiatry 29, 61-67.

Van den Broek, M. D., Bradshaw, C. M., and Szabadi, E. (1993). Utility of the Modified Wisconsin Card Sorting Test in neuropsychological assessment. Br. J. Clin. Psychol. 32(Pt 3), 333-343.

Van der Werf, Y., Scheltens, P., Lindeboomc, J., Witter, M. P., Uylings, H. B., and Jolles, J. (2003). Deficits of memory, executive functioning and attention following infarction in the thalamus; a study of 22 cases with localized lesions. Neuropsychologia 41, 1330-1344.

Whyte, E., Lenze, E., Butters, M., Skidmore, E., Koenig, K., Dew,
M., Penrod, L., Mulsant, B., Pollock, B., Cabacungan, L., Reynolds, C., and Munin, M. (2008). An open-label pilot study of acetylcholinesterase inhibitors to promote functional recovery in elderly cognitively impaired stroke patients. Cerebrovasc. Dis. 26, 317-321.

Wilson, B. A., Evans, J., Emslie, H., Alderman, N., and Burgess, P. W. (1998). The development of an ecologically valid test for assessing patients with a dysexecutive syndrome. Neuropsychol. Rehabil. 8, 213-228.

Conflict of Interest Statement: The authors declare that the research was conducted in the absence of any commercial or financial relationships that could be construed as a potential conflict of interest.

Received: 31 October 2010; accepted: 07 March 2011; published online: 24 March 2011.

Citation: Riveros $R$, Chabriat H, Flores $R$, Alvarez $G$ and Slachevsky A (2011) Effects of donepezil on behavioral manifestations of thalamic infarction: a singlecase observation. Front. Neur. 2:16. doi: 10.3389/fneur.2011.00016

This article was submitted to Frontiers in Dementia, a specialty of Frontiers in Neurology.

Copyright (C) 2011 Riveros, Chabriat, Flores, Alvarez and Slachevsky. This is an open-access article subject to an exclusive license agreement between the authors and Frontiers Media SA, which permits unrestricted use, distribution, and reproduction in any medium, provided the original authors and source are credited. 